\title{
A Class of Stable Algorithms for Stiff Ordinary Differential Equation System
}

\author{
Ying-Qiu Gu \\ School of Mathematical Science, Fudan University, Shanghai 200433, China \\ Email: yqgu@fudan.edu.cn
}

\begin{abstract}
In this paper, we introduce a series of stable algorithms for solving the stiff ordinary differential equation system. These algorithms are based on the solution to the local linearized perturbation equation and Padé approximations of exponential function. The algorithms get rid of the influence of the stiffness and have explicit schemes. In contrast with conventional implicit schemes, this class of schemes has some advantages such as the simple program code, high precision, good convergence and strong stability by the virtue of Padé approximation. It is a good assistant for the researchers unfamiliar with the numerical analysis theory.
\end{abstract}

Keywords: Stiff ordinary differential equation system, Padé approximant, stable algorithm, explicit algorithm

\section{Introduction}

In scientific research and industry such as celestial mechanics, weather prediction, biology, thermonuclear reaction, automatic control, electronic network and chemical kinetics, the physical and chemical processes are often described by ordinary differential equations(ODE). The variables in these processes often have very different rates of change, that is, the solutions to the ordinary differential equation system include both fast decreasing components and slowly changing ones. When we solve the numerical solutions of such kind of ordinary differential equations on a large time scale, the fast decreasing components can be soon ignored. But these fast decreasing components will seriously interfere with the accuracy and stability of the numerical solutions of the whole differential equations, and bring great difficulties to the actual calculation, which is called stiff problem.

The definition of stiff ODE was studied in $[1,2]$. For the following ODE

$$
y^{\prime}=f(y), \quad y(0)=y_{0}, \quad\left(y \in R^{N}\right),
$$

the definition of stiffness is currently accepted as follows. If the solution to (1) satisfies two conditions, (i) the solution changes slowly over $t \in[a, b]$; (ii) for a solution of $y(t),(\forall t \in[a, b])$, the characteristic values of Jacobian matrix $\partial_{y} f(y)$ have no large positive real part, but have at least one large negative real part, then the system is called stiff ODE.

In the numerical simulation for ODE (1), we have general method with step $h$

$$
\sum_{j=0}^{k} \alpha_{j} y_{n+j}=h \varphi\left(y_{n}, y_{n}+1, \cdots, y_{n+k} ; h\right), \quad(n=0,1, \cdots, N-k) .
$$

The explicit schemes such as linear multi-step methods and Runge-Kutta methods are unstable and divergent for stiff problem, the algorithms for the stiff ODE must be implicit algorithms, and the order of precision of the stable algorithms is limited[3,4,5,6]. The algorithms (2) include constructing and solving nonlinear algebraic equations, which involve some complicated conditions and skills for the programming and stability of the algorithms.

Solving the implicit one-step methods for the linear test equation

$$
y^{\prime}=\lambda y, \quad y(0)=y_{0}, \quad\left(y, \lambda \in C^{1}, \Re(\lambda)<0\right),
$$


we get the difference scheme

$$
y_{n+1}=R_{j, k}(\lambda h) y_{n}
$$

where $y_{n}$ stands for the numerical solution of $y(n h), R_{j, k}$ is a $j+k$ degree rational approximation of $e^{\lambda h}$. If this approximation is also $j+k$ order, $R_{j, k}$ becomes the Padé approximation of $e^{\lambda h}[6,7]$,

$$
R_{j, k}(z)=G_{j}(k,-z)^{-1} G_{k}(j, z), \quad G_{k}(j, z) \equiv \sum_{m=0}^{k} \frac{(j+k-m) ! k !}{(j+k) ! m !(k-m) !} z^{m}
$$

The Padé approximation (5) has the following properties:

$\mathbf{P 1}$. The remainder term is given by

$$
e^{z}=R_{j, k}(z)+\frac{(-1)^{k} j ! k !}{(j+k) !(j+k+1) !} z^{j+k+1}+\mathrm{O}\left(z^{j+k+2}\right) .
$$

P2. $R_{j, k}$ is A-acceptable iff $k \leq j \leq k+2$, and L-acceptable iff $k+1 \leq j \leq k+2$, where the A-(L-)acceptable means the difference scheme (4) is A-(L-)stable[8,9,10,11,12].

P3. If $j \leq k+4$, then $R_{j, k}$ has no poles in the left plane $\{z \mid \Re(z) \leq 0\}$.

P4. In the imaginary axis $z=y \mathrm{i},\left|R_{j, j}(z)\right| \equiv 1[13]$.

The above properties are excellent for solving the numerical solution of stiff ODE. For example, in $[14,15,16]$, a class of symplectic schemes for Hamilton-Jacobian system is constructed from $R_{j, j}$, which can hold some important features of the original system such as some conserved quantities. The following analysis shows that, the stiffness of the equation system (1) is mainly described by its locally linearized equation, and the nonlinear remainder is only a third order perturbation independent of stiffness. Applying the Padé approximation to the solution of this linearized perturbational equation, we can get rid of the influence of the stiffness and derive a series of explicit algorithms with simple program code, high order precision, good convergence and strong stability.

\section{Construction of the Difference Schemes}

For the general stiff ODE (1), suppose $f(y)$ is smooth enough. Denoting

$$
f_{0}=f\left(y_{0}\right), \quad J_{0}=\frac{\partial}{\partial y} f\left(y_{0}\right), \quad u=y-y_{0},
$$

then (1) can be rewritten as locally linearized equation plus a perturbation

$$
u^{\prime}=f_{0}+J_{0} u+\Delta(u), \quad u(0)=0,
$$

where

$$
\Delta(u) \equiv f\left(y_{0}+u\right)-f_{0}-J_{0} u
$$

If $\left\|\partial_{y}^{2} f(y)\right\| \leq 2 c$ in the neighborhood of $u_{0}$, then we have

$$
\|\Delta(u)\| \leq c\|u\|^{2}
$$

where $\|\cdot\|$ is any vector norm or the corresponding induced norm for derivative operators.

If we treat $\Delta(u(t))$ as a known function of $t$ in (8), then the solution $u(t)$ can be formally represented by the following integral

$$
u(t)=\left[J_{0}^{-1}\left(e^{J_{0} t}-I\right)\right] f_{0}+\int_{0}^{t} e^{J_{0}(t-\tau)} \Delta(u(\tau)) d \tau
$$

If $J_{0}$ is singular, it is treated as $J_{0}+\epsilon I$ as $\epsilon \rightarrow 0$ in the matrix $\left[J_{0}^{-1}\left(e^{J_{0} t}-I\right)\right]$. 
The formal solution (11) has a wonderful advantage, that is, the coefficient matrix $e^{J_{0}(t-\tau)}$ is a strong contractive operator for stiff problem, so the high order implicit scheme can be efficiently solved by iteration. In the usual cases, we can use interpolation polynomial of $t$ to approximate $\Delta(u(t))$ in the neighborhood of $t=0$. By (10), we have

$$
\Delta(u(t))=\left(A_{0}+A_{1} t+\cdots+A_{p-3} t^{p-3}\right) t^{2}+\mathrm{O}\left(t^{p}\right),
$$

where $\left\{A_{l}\right\}$ are the interpolation coefficient vectors. By

$$
\int_{0}^{t} e^{J_{0}(t-\tau)} \cdot \tau^{l} d \tau=l ! J_{0}^{-(l+1)}\left[e^{J_{0} t}-\sum_{m=0}^{l} \frac{1}{m !}\left(J_{0} t\right)^{m}\right],
$$

substituting (12) and (13) into (11) we get

$$
u(t)=J_{0}^{-1}\left(e^{J_{0} t}-I\right) f_{0}+\sum_{l=0}^{p-3} A_{l}(l+2) ! J_{0}^{-(l+3)}\left[e^{J_{0} t}-\sum_{s=0}^{l+2} \frac{1}{s !}\left(J_{0} t\right)^{s}\right]+\mathrm{O}\left(t^{p+1}\right) .
$$

We express $e^{J_{0} t}$ by $p=j+k$ order A- or L-acceptable Padé approximation

$$
e^{J_{0} t}=R_{j, k}\left(J_{0} t\right)+\mathrm{O}\left(t^{p+1}\right), \quad R_{j, k}=P_{j, k} Q_{j, k}^{-1} .
$$

Substituting it into (14) and Omitting $\mathrm{O}\left(t^{p+1}\right)$ terms, we have

$$
\begin{aligned}
Q_{j, k} u(t)= & J_{0}^{-1}\left(P_{j, k}-Q_{j, k}\right) f_{0}+ \\
& \sum_{l=0}^{p-3}(l+2) !\left(J_{0}\right)^{-(l+3)}\left[P_{j, k}-Q_{j, k} \sum_{s=0}^{l+2} \frac{1}{s !}\left(J_{0} t\right)^{s}\right] A_{l} .
\end{aligned}
$$

Converting (16) into difference scheme from $t_{n}$ to $t_{n+1}$, we get $p$-th order scheme as follows

$$
\begin{aligned}
Q_{j, k} y_{n+1}= & Q_{j, k} y_{n}+T^{-1}\left(P_{j, k}-Q_{j, k}\right) f_{n} h+ \\
& \sum_{l=0}^{p-3}(l+2) ! T^{-(l+3)}\left(P_{j, k}-Q_{j, k} \sum_{s=0}^{l+2} \frac{1}{s !} T^{s}\right) A_{l} h^{l+3},
\end{aligned}
$$

in which $y_{n}$ is the numeric solution of $y\left(t_{n}\right)$ and

$$
\left\{\begin{array}{l}
u_{n+1}=y_{n+1}-y_{n}, \quad h=t_{n+1}-t_{n} \\
f_{n}=f\left(y_{n}\right), \quad T=J_{n} h=\frac{\partial}{\partial y} f\left(y_{n}\right) h
\end{array}\right.
$$

For implicit scheme, the interpolation coefficient $A_{l}$ depends on $y_{n+1}$. By $y_{n+1}, y_{n}, \cdots, y_{n-(p-3)}, A_{l}$ for the $p$-th schemes can be calculated as follows,

$$
\Delta(u(t))=\sum_{l=n-p+3}^{n+1} F_{l} \frac{t-t_{n}}{t_{l}-t_{n}} \prod_{k=n-p+3, k \neq l}^{n+1} \frac{t-t_{k}}{t_{l}-t_{k}}+\mathrm{O}\left(t-t_{n}\right)^{p},
$$

where

$$
F_{l}=f_{l}-f_{n}-J_{n}\left(y_{l}-y_{n}\right), \quad\left(F_{n}=0\right)
$$

For linear ODE, (17) becomes the pure Padé approximation of exponential functions. By the property $\mathrm{P} 2$, the scheme (17) is A-stable if $j=k$, and L-stable if $k+1 \leq j \leq k+2$. By the property $\mathrm{P} 3, Q_{j, k}$ is invertible for all $r h<1$, where

$$
r \equiv \max _{\forall l}\left\{\Re\left(\lambda_{l}\right)\right\}
$$


$\lambda_{l}$ is the eigenvalue of $J_{n}$. By (20) we find $r$ is independent of the stiffness, because the stiffness is determined by $\lambda_{l}$ with large negative real part.

Now we construct some concrete schemes. The second order schemes are all explicit, because the nonlinear term $\Delta(u)$ is a third order perturbation as shown below. For second order A-stable scheme, by

$$
R_{1,1}(z)=P_{1,1} Q_{1,1}^{-1}=\left(1+\frac{1}{2} z\right)\left(1-\frac{1}{2} z\right)^{-1}
$$

substituting it into (17) we get

$$
\left(I-\frac{1}{2} T\right)\left(y_{n+1}-y_{n}\right)=f_{n} h
$$

For the 2-order L-stable scheme

$$
R_{2,0}(z)=\left(1-z+\frac{1}{2} z^{2}\right)^{-1}
$$

by (17) we have

$$
\left(I-T+\frac{1}{2} T^{2}\right)\left(y_{n+1}-y_{n}\right)=\left(I-\frac{1}{2} T\right) f_{n} h .
$$

For the third order L-stable scheme,

$$
R_{2,1}(z)=\left(1+\frac{1}{3} z\right)\left(1-\frac{2}{3} z+\frac{1}{6} z^{2}\right)^{-1} .
$$

By (19), we get the second order interpolation polynomial

$$
\Delta(u(t))=\left[f_{n+1}-f_{n}-J_{n} u_{n+1}\right]\left(\frac{t-t_{n}}{h}\right)^{2}+\mathrm{O}\left(h^{3}\right) .
$$

Substituting (25) and (26) into (17) we get a third order scheme

$$
\left(I-\frac{2}{3} T+\frac{1}{6} T^{2}\right) u_{n+1}=\left(I-\frac{1}{6} T\right) f_{n} h+\frac{1}{3}\left(I-\frac{1}{2} T\right)\left[f_{n+1}-f_{n}-J_{n} u_{n+1}\right] h .
$$

Similarly, we can easily construct higher order schemes. In these schemes, the solved values $y_{n}, y_{n-1}, y_{n-2}, \cdots$ are used to interpolate $\Delta(u(t))$ and the schemes become multi-step methods. Obviously the order of precision of the scheme (17) is not limited.

\section{Convergence of the Algorithm}

Now we discuss the convergence and stability of the schemes. We take the following hypotheses for convenience, but the conclusions can be easily generalized.

H1. There is a $U$-matrix $U$, such that

$$
U J_{0} U^{-1}=\operatorname{diag}\left(\lambda_{1}, \lambda_{2}, \cdots, \lambda_{N}\right)
$$

H2.

$$
r=\max _{\forall k}\left\{\Re\left(\lambda_{k}\right)\right\} \leq 0
$$

H3. There exists $R>0$, such that the second order differential operator of $f(y)$ is bounded in the domain $\left\|y-y_{0}\right\| \leq R$, namely

$$
\max _{\forall\left\|y-y_{0}\right\| \leq R}\left\|\partial_{y}^{2} f(y)\right\|=2 c
$$


where the norm and the inner product of vectors are defined by

$$
\|x\| \equiv \sqrt{x^{+} x}, \quad(x, y) \equiv x^{+} y,
$$

and the norm of the derivative operators $\partial_{y} f(y), \partial_{y}^{2} f(y)$ are the corresponding induced norm, where the index '+' denotes transposed conjugate.

Theorem 1. Assume that $v$ is the solution of linearized equation

$$
v^{\prime}=f_{0}+J_{0} v, \quad v(0)=0,
$$

$u$ is the solution of (8). If H1, H2 and H3 hold, then we have

$$
\|u-v\| \leq p(t)-\frac{1}{r}\left(e^{r t}-1\right)\left\|f_{0}\right\|,
$$

where

$$
p(t)=\left\{\begin{array}{lll}
\frac{2 \tan (\alpha t)\left\|f_{0}\right\|}{2 \alpha-r \tan (\alpha t)} & \text { if } & r^{2}<4 c\left\|f_{0}\right\|, \\
\frac{2 t\left\|f_{0}\right\|}{2-r t} & \text { if } & r^{2}=4 c\left\|f_{0}\right\|, \\
\frac{2 \tanh (\alpha t)\left\|f_{0}\right\|}{2 \alpha-r \tanh (\alpha t)} & \text { if } & r^{2}>4 c\left\|f_{0}\right\|,
\end{array}\right.
$$

in which $\alpha=\frac{1}{2} \sqrt{\left|r^{2}-4 c\right|\left|f_{0}\right| \mid}$.

Proof. By (32) and (H1,H2), we have

$$
\begin{aligned}
\frac{d}{d t}\|v\|^{2} & =\left(v, f_{0}\right)+\left(f_{0}, v\right)+(U v)^{+}\left[\left(U J_{0} U^{-1}\right)^{+}+\left(U J_{0} U^{-1}\right)\right](U v) \\
& \leq 2\|v\| \cdot\left\|f_{0}\right\|+2 r\|v\|^{2}, \quad(\|v(0)\|=0) .
\end{aligned}
$$

By Gronwall inequality we get

$$
\|v\| \leq \frac{1}{r}\left(e^{r t}-1\right)\left\|f_{0}\right\| \equiv q(t)
$$

where

$$
q^{\prime}=\left\|f_{0}\right\|+r q, \quad q(0)=0 .
$$

Let $w=u-v$, by (8) and (32) we have

$$
w^{\prime}=J_{0} w+\Delta(w+v), \quad w(0)=0 .
$$

By $\mathrm{H} 1$ and $\mathrm{H} 3$ we have

$$
\begin{aligned}
\frac{d}{d t}\|w\|^{2} & \leq 2 r\|w\|^{2}+2 c\|w\|(\|w\|+\|v\|)^{2} \\
& \leq 2 r\|w\|^{2}+2 c\|w\|[\|w\|+q(t)]^{2}, \quad\|w(0)\|=0
\end{aligned}
$$

or equivalently

$$
\frac{d}{d t}\|w\| \leq r\|w\|+c[\|w\|+q(t)]^{2}, \quad\|w(0)\|=0 .
$$

For the following equation

$$
p^{\prime}=\left\|f_{0}\right\|+r p+c p^{2}, \quad p(0)=0,
$$

the solution is given by (34). Denoting $W=p-q$, by (37) and (41), we have

$$
W^{\prime}=r W+c[W+q(t)]^{2}, \quad W(0)=0 .
$$


Comparing (40) with (42), we get $\|w\| \leq W=p-q$. By (34) and (36), the proof is finished.

The above theorem shows how the parameters $(r, c)$ influence precision of the linearized equation. By (33) and (34), we get

$$
\|u-v\| \leq \frac{1}{3} c\left\|f_{0}\right\|^{2} t^{3}+\mathrm{O}\left(t^{4}\right)
$$

so the nonlinear term $\Delta(u)$ only results in a third order perturbation to the solution, and the perturbation is independent of stiffness of the ODE. In what follows, we take scheme (27) as an example to show the convergence and stability.

Lemma 2. If H1, H2 and H3 hold, and the step length $h$ satisfies

$$
h \leq \widetilde{h} \equiv \sqrt{\frac{3}{4 c\left\|f_{0}\right\|}},
$$

then the sequence $\left\{X_{m} \mid m=0,1,2, \cdots\right\}$ generated by the iterative scheme of (27)

$$
X_{m+1}=A f_{0} h+B\left[f\left(y_{0}+X_{m}\right)-f_{0}-J_{0} X_{m}\right] h, \quad X_{0}=0
$$

is bounded, where

$$
\left\{\begin{array}{l}
A=\left(I-\frac{2}{3} T+\frac{1}{6} T^{2}\right)^{-1}\left(I-\frac{1}{6} T\right), \quad T=J_{0} h \\
B=\frac{1}{3}\left(I-\frac{2}{3} T+\frac{1}{6} T^{2}\right)^{-1}\left(I-\frac{1}{2} T\right)
\end{array}\right.
$$

Proof. By $\mathrm{H} 1$ and $\mathrm{H} 2$, it is easy to check

$$
\|A\| \leq 1, \quad\|B\| \leq \frac{1}{3} .
$$

By (45) and H3, we have

$$
\left\|X_{m+1}\right\| \leq\left\|f_{0}\right\| h+\frac{1}{3} c\left\|X_{m}\right\|^{2} h, \quad\left\|X_{0}\right\|=0
$$

If (44) holds, the following monotone increasing sequence $\left\{a_{m}\right\}$

$$
a_{m+1}=\left\|f_{0}\right\| h+\frac{1}{3} \operatorname{ch} \widetilde{a} a_{m}, \quad a_{0}=0
$$

is bounded, and we have

$$
a_{m} \rightarrow \widetilde{a} \equiv \frac{3}{2 c h}\left(1-\sqrt{1-\frac{4}{3} c\left\|f_{0}\right\| h^{2}}\right) \leq \sqrt{\frac{3\left\|f_{0}\right\|}{c}} .
$$

Comparing (48) with (49), by induction we can prove $\left\|X_{m}\right\| \leq a_{m}<\widetilde{a},(\forall m)$. This finishes the proof.

The theorem shows this scheme is stable if $h \leq \widetilde{h}$, and $\widetilde{h}$ is irrelative to the stiffness.

Theorem 3. Under the conditions of Lemma 2, the iterative scheme (3.17) is convergent.

Proof. By (45) we get

$$
\left\|X_{m+1}-X_{m}\right\| \leq h\|B\| \cdot\left\|f\left(y_{0}+X_{m}\right)-f\left(y_{0}+X_{m-1}\right)-J_{0}\left(X_{m}-X_{m-1}\right)\right\| .
$$

By mean value theorem and (47), we have

$$
\left\|X_{m+1}-X_{m}\right\| \leq \frac{1}{3} h\left\|\partial_{y}^{2} f\right\| \cdot\|\xi\| \cdot\left\|X_{m}-X_{m-1}\right\| .
$$

By Lemma 2 we learn $\|\xi\| \leq \widetilde{a}$, so

$$
\left\|X_{m+1}-X_{m}\right\| \leq \delta\left\|X_{m}-X_{m-1}\right\|,
$$


where the contractive coefficient

$$
\delta \equiv 1-\sqrt{1-\frac{4}{3} c|| f_{0} \| h^{2}}<1 .
$$

The proof is finished.

Theorem 3 shows that the nonlinear scheme (27) can be solved by direct iteration if $h$ is controlled by (44). Further analysis shows that, the parameter $\mu_{n} \equiv \sqrt{c\left\|f_{n}\right\|}$ is a measurement for nonlinearity, which is invariant under the scaling transformation $z=\kappa y$. If $\mu_{n} h \ll 1$, the solution is mainly determined by the linear part of the equation.

Theorem 4. The schemes (17) keep the linear conserved quantities of the original equation (1).

Proof. Suppose

$$
V(t)=\sum_{l=1}^{N} C_{l} Y_{l}(t)=C \cdot y(t)
$$

is conserved, i.e. $V(t) \equiv V(0)$, where $C \equiv\left(c_{1}, c_{2}, \cdots, c_{N}\right)$ is the coefficient vector, $Y_{l}(t)$ is the $l$-th element of $y$. Since

$$
0=V^{\prime}=C \cdot y^{\prime}=C \cdot f(y)
$$

we have

$$
C \cdot \frac{\partial f}{\partial y}=C \cdot J(y) \equiv 0 .
$$

Multiplying (17) by $C$, and using (56) and (57) repeatedly, by $C \cdot f_{n}=0, C \cdot T=0$, we get

$$
C \cdot Q_{j, k} u_{n+1}=C \cdot u_{n+1}=C \cdot\left(y_{n+1}-y_{n}\right)=0,
$$

so $V_{n+1}=V_{n}$. The proof is finished.

\section{$4 \quad$ Numerical Experiments}

At first, we examine the scheme (27) by the nonlinear test equation

$$
\begin{array}{rlrl}
Z^{\prime} & =(\lambda-Z) Z, & Z(0) & =Z_{0}, \\
J & =f^{\prime}=\lambda-2 Z, & f^{\prime \prime}=-2,
\end{array}
$$

where $\left(\lambda, Z, Z_{0}\right) \in \mathcal{C}^{1}, \Re(\lambda) \leq 0$. The rigorous solution is given by

$$
Z(t)=\frac{\lambda Z_{0}}{Z_{0}+\left(\lambda-Z_{0}\right) e^{-\lambda t}} .
$$

In the case $\Re(\lambda)<0$, all the trajectories start from $Z=\lambda$ as $t \rightarrow-\infty$ and converge to $Z=0$ as $t \rightarrow \infty$. $\Re(\lambda)=0$ corresponds to periodic trajectory.

In numeric simulation, we set $Z=X+Y \mathrm{i}$ in (59) to transform it into the real form, then we get

$$
\begin{aligned}
& X^{\prime}=u X-v Y-X^{2}+Y^{2}, \\
& Y^{\prime}=v X+u Y-2 X Y,
\end{aligned}
$$

in which $\lambda=u+v$ i.

Denote the numerical solution by $(x, y)$. From FIG.1, we find that, the coefficient functions of error $\left|(X-x) / h^{3}\right|$ and $\left|(Y-y) / h^{3}\right|$ approach limit functions as $h \rightarrow 0$, so the scheme (27) has global third order precision. On the other hand, these functions are decreasing functions of $h$, which means the scheme is strongly stable. However, the absolute values of coefficient functions are quite large, which is caused by $\lambda$. 


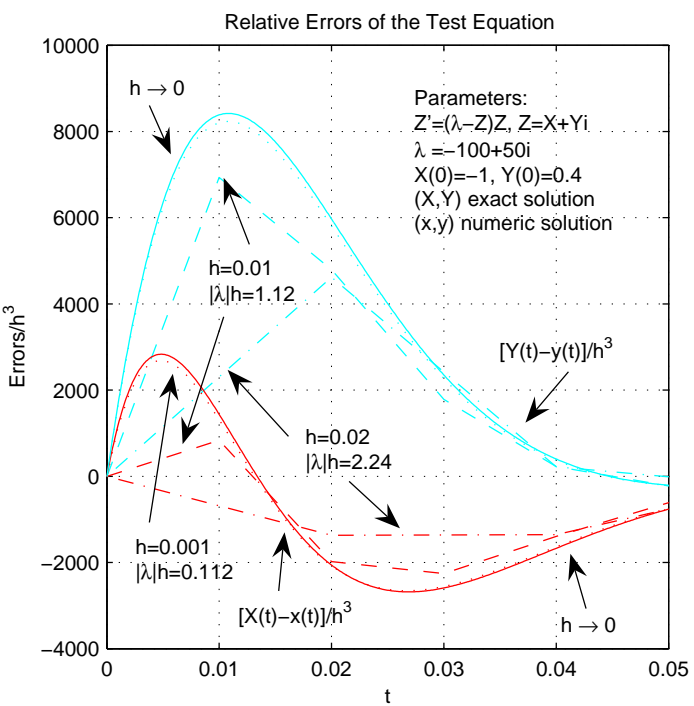

Figure 1. The coefficient functions of errors $(X-x) / h^{3}$ and $(Y-y) / h^{3}$, which show the scheme (27) has third order global precision and strong stability.

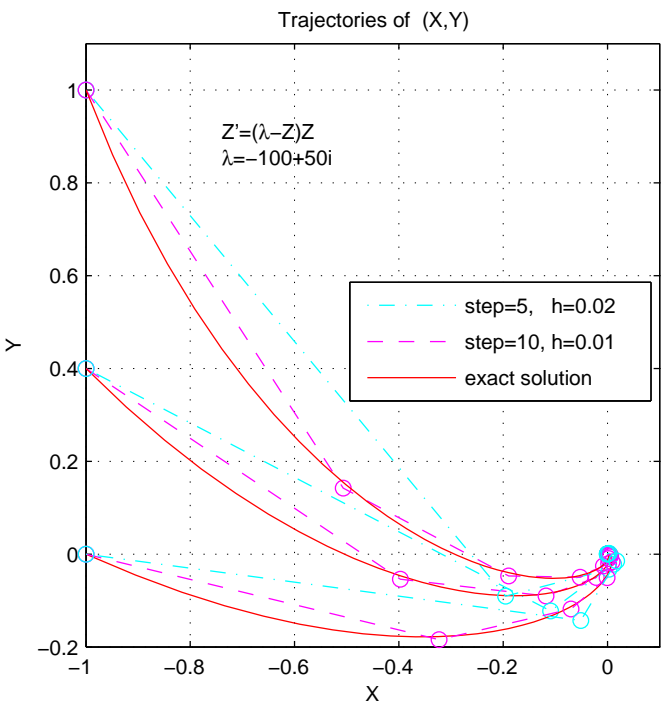

Figure 2. The exact and numerical trajectories of the ODE $Z^{\prime}=(\lambda-Z) Z$. Simulation shows the scheme (27) is strong stable even if $|\lambda h| \gg 1$.

The exact and numerical trajectories of the nonlinear test equation (62) and (63) are displayed in FIG.2, which shows the numerical solutions keep the main features of the exact solutions.

At last, we solve the following chemical reaction equation system

$$
\left\{\begin{array}{l}
y_{1}^{\prime}=-0.04 y_{1}+y_{2} y_{3} \\
y_{2}^{\prime}=400 y_{1}-10^{4} y_{2} y_{3}-3 \times 10^{3} y_{2}^{2} \\
y_{3}^{\prime}=0.3 y_{2}^{2}, \\
y_{1}(0)=1, \quad y_{2}(0)=y_{3}(0)=0
\end{array}\right.
$$

The eigenvalues of the Jacobian matrix $J$ are $\left(\lambda_{1}, \lambda_{2}, \lambda_{3}\right)=(0,0,-0.04)$ at $t=0$, but they becomes $\left(\lambda_{1}, \lambda_{2}, \lambda_{3}\right)=(0,-0.36,-2180)$ at $t=0.01$. This means the dynamical equation system has not only high stiffness but also large $\left\|\partial_{y}^{2} f\right\|$ in the neighborhood of $t=0$. The maximum step length for convergence of iteration at $t=0$ is $h_{\max } \doteq 0.00136$, but $h_{\max }>3.5$ if $t>0.001$. So we use the adaptive step length in the numerical simulation. The numerical result is displayed in FIG.3, which shows $y_{2}(t)$ increases rapidly near $t=0$. (64) has a linear conserved quantity $y_{1}(t)+10^{-4} y_{2}(t)+y_{3}(t) \equiv 1$. The numerical solution also exactly keeps this relation.

In summary, expressed the solution of the local linearized perturbation equation of the stiff ODE by Padé approximations of exponential function, we introduce a series of algorithms to resolve the numerical solution. The algorithms get rid of the influence of the stiffness and have high order schemes. In contrast with conventional implicit schemes, the above schemes have simple program code, high order precision, good convergence and strong stability. They are good assistant of the researchers unfamiliar with the numerical analysis theory.

Acknowledgments. The author is grateful to the supervisors Prof. Ta-tsian Li and Prof. Han-Ji Shang for their encouragement.

\section{References}

1. J. D Lambert, Computational Methods in Ordinary Differential Equations, John Wiley \& Sons Ltd., 1973 


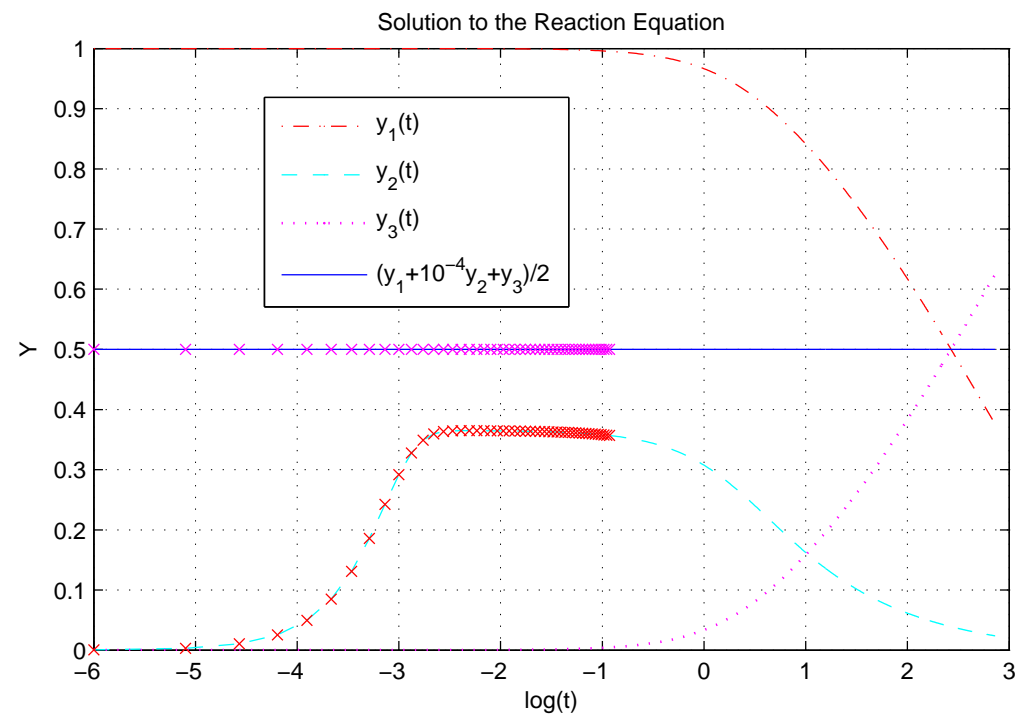

Figure 3. The solution of the reaction equation (64), $y_{2}$ increases rapidly near $t=0$.

2. L. F. Shampine, C. W. Gear. A User's View of Solving Stiff Ordinary Differential Equations. SIAM Review, 1979, 21(1):1-17.

3. G. Dahlquist, Convergence and stability in the numerical integration of ordinary differential equations. Math. Scand. $4,33-53$ (1956)

4. O. B. Widlund, A note on unconditionally stable linear multistep methods. BIT, 1967, 7(1):65-70.

5. Li Shoufu, Ruan Baogen, Nonlinear Stability of Multistep Multiderivative Methods, Mathematics of Computation, 1990, 55(192):581-589.

6. G. Wanner, E. Hairer, P. Norsett, Order star and stability theorems, BIT18(4),475-489(1978),

7. Xu Xian-Yu, Li Jia-Kai, Xu Guo-Liang, General theory of Padé approximation(in Chinese), Shanghai Scientific and Technology Press, 1990

8. B. L. Ehle, On Padé approximations to the exponential function and A-stable methods for the numerical solution of initial value problems, Univ. of Waterloo, Rep. No.CSRR 2010, 1969

9. E. B. Saff, R. S. Varga, On the zeros and poles of Padé approximants to $e^{x}$, Numer. Math. 25(1), 1-4(1975)

10. E. B. Saff, R. S. Varga, Zero-free parabolic region for sequences of polynomials, SIAM J. Math. Anal. 7(3), 344-357(1976)

11. S. P. N $\phi$ rsett, C-polynomials for rational approximation to the exponential function, Numer. Math. 25(1), 39-56(1975)

12. E. Hairer, G. Wanner, Solving ordinary differential equations II - stiff and differential algebraic problems, Springer, Berlin, 1996

13. J. L. Gammel, F. A. McDonald, Applications of the Padé approximation to scattering theory, Phys. Rev. $142,1245-1254(1966)$

14. Feng Kang, Difference schemes for Hamiltonian formalism and symplectic geometry, J. Comp. Math. 4(3), 279-289(1986)

15. Feng Kang, et al, Construction of canonical difference schemes for Hamiltonian formalism via generating function, J. Comp. Math. 7(1), 72-96(1989)

16. Feng Kang, Wang Dao-Liu, Symplectic Difference schemes for Hamiltonian system in general symplectic structure, J. Comp. Math. 9(1), 86-96(1991) 